\title{
Super-High Temperature Sensitivity of Long-Period Gratings in B/Ge Co-doped Fiber
}

\author{
Xuewen Shu*, Bashir A.L. Gwandu, Lin Zhang, Ian Bennion \\ Photonics Research Group, Aston University, Birmingham, B47ET, United Kingdom
}

\begin{abstract}
Long period fiber grating (LPFG) can be used as active gain controlling device in EDFA. However, LPFGs fabricated in the standard telecom fiber only have a typical temperature sensitivity of $3-10 \mathrm{~nm} / 100^{\circ} \mathrm{C}$, which may not be sufficient for implementing tuneable filters capable of wide tuning range and high tuning efficiency. In this paper, we report a theoretical and experimental investigation of thermal properties of LPFGs fabricated in B/Ge co-doped optical fiber. We have found that the temperature sensitivity of the LPFGs in the B/Ge fiber is considerably increased compared with those produced in the standard fiber. The LPFGs written in the B/Ge fiber have achieved, on average, one order of magnitude higher sensitivity than that of the LPFGs produced in the standard telecom fiber. We have also identified that the thermal response of LPFG is strongly dependent on the order of the coupled resonant cladding mode. The maximum sensitivity of $1.75 \mathrm{~nm} /{ }^{\circ} \mathrm{C}$ achieved by the $10^{\text {th }}$ cladding mode of the $240 \mu \mathrm{m}$ LPFG is nearly 24 times that of the minimum value $(0.075 \mathrm{~nm} / \mathrm{C})$ exhibited by the $30^{\text {th }}$ mode of the $34 \mu \mathrm{m}$ LPFG. Such devices may lead to high-efficiency and low-cost thermal/electrical tunable loss filters or sensors with extremely high temperature resolution.
\end{abstract}

Keywords: Long period fiber grating, fiber Bragg grating, grating, EDFA, thermal coefficient, fiber optical sensor

\section{INTRUDUCTION}

Long-period fiber gratings (LPFGs) offer a number of distinct advantages, including easy fabrication, low level of backreflection and high temperature sensitivity, which render them more desirable for some special applications. Recently, several types of LPFG-based thermal or electrical tuneable filters have been demonstrated for high-performance active gain controlling of EDFA systems. However, LPFGs fabricated in the standard telecom fiber only have a typical temperature sensitivity of $3-10 \mathrm{~nm} / 100^{\circ} \mathrm{C}[1,2]$, which may not be sufficient for implementing tuneable loss filters capable of wide tuning range and high tuning efficiency. It has been demonstrated that the temperature sensitivity of LPFG can be significantly enhanced by recoating or partially cladding the fiber with special polymers [3-5]. In this paper, we report a theoretical and experimental investigation of thermal properties of LPFGs fabricated in the conventional B/Ge co-doped fiber. We have found that the temperature sensitivity of the LPFGs in the B/Ge fiber is considerably increased compared with those produced in the standard fiber. We have also identified that the thermal response of LPFG is strongly dependent on the order of the coupled resonant cladding mode. These findings offer a useful guidance for designing LPFG devices of low- or high-temperature sensitivity.

\section{THEORY}

The temperature sensitivity of resonance mode of the LPFG can be expressed as

\footnotetext{
*X.Shu@aston.ac.uk; phone 441213593621 5253; fax: 44121 3590156; Photonics Research Group, Aston University, Birmingham, B47ET, United Kingdom. Xuewen Shu is now with Indigo Photonics Ltd. Faraday Wharf, Holt Street, Birmingham, B7 4BB, United Kingdom
} 


$$
\frac{d \lambda_{\text {res }}}{d T}=\lambda_{\text {res }} \cdot \gamma \cdot(\alpha+\Gamma)
$$

where $\gamma$ describes the waveguide dispersion and $\Gamma$ describes the temperature dependence of the waveguide dispersion. They are defined as

$$
\gamma=\frac{\frac{d \lambda_{r e s}}{d \Lambda}}{n_{c o}^{e f f}-n_{c l, m}^{e f f}} \quad \text { and } \quad \Gamma=\frac{\xi_{\mathrm{co}} n_{c o}^{e f f}-\xi_{\mathrm{cl}} n_{c l, m}^{e f f}}{n_{c o}^{e f f}-n_{c l, m}^{e f f}}
$$

where $\xi_{c o}$ and $\xi_{c l}$ are the thermo-optic coefficients of the core and cladding material, respectively, and $\alpha$ is the thermal expansion coefficient of the fiber. In general, $\alpha<<\Gamma$ is true for the silica-based fibers. According to eqn. 1, the temperature sensitivity is predominately determined by the term of the product $\gamma \cdot \Gamma$. Using the weakly guiding approximation method [6], we calculated the $\gamma$ and $\Gamma$ for a set of LPFGs of various periods for $\lambda_{\text {res }}=1500 \mathrm{~nm}$ and the results versus the first thirty modes are plotted in fig. 1. It is interesting to know from this figure that $\gamma$ appears to have large values and changes sign from positive to negative in the region near the $10^{\text {th }}$ and $11^{\text {th }}$ modes, while $\Gamma$ always remains negative and $|\Gamma|$ decreases with the mode order. It is thus clear that the sign combination of $\gamma$ and $\Gamma$ will lead to positive temperature coefficients for the modes of high orders $(\mathrm{m} \geq 11)$ and negative coefficients for the modes of low orders $(\mathrm{m} \leq 10)$. The magnitude combination of $\gamma$ and $\Gamma$ will lead to two maximums of $|\gamma \cdot \Gamma|$ for $10^{\text {th }}$ and $11^{\text {th }}$ modes, consequently giving two super-high temperature sensitivity modes. Although the calculation was for a defined wavelength, the operation wavelengths of these two sensitive modes can be tailored by changing the period size of the grating. Hence, it is possible to design LPFG possessing a cladding mode in the required operation wavelength range with the order close to the sensitive regime.

\section{EXPERIMENT AND RESULTS}

In order to experimentally investigated the dependence of the thermal response on the cladding mode order, seven LPFGs with small, medium and large periods were UV inscribed using amplitude masks and a pulsed frequency quadrupled YAG laser in the conventional $\mathrm{B} / \mathrm{Ge}$ co-doped fiber without hydrogenation. After exposure, all the gratings were annealed to stabilise their optical properties. Then, their spectral profiles were examined at room temperature using a broadband LED and an optical spectrum analyser (OSA) with a resolution of $0.1 \mathrm{~nm}$. The resonant wavelengths of these modes with their corresponding mode orders and grating periods are listed in table 1.

The thermal response of the LPFGs was characterised by heating them from $0^{\circ} \mathrm{C}$ to $60^{\circ} \mathrm{C}$ with an incremental step of $10^{\circ} \mathrm{C}$ using a temperature controllable thermoelectric cooler. The resonance wavelength shifts against temperature for all the seven LPFGs are plotted in fig.2, showing two interesting principal trends which are in excellent agreement with the theoretical analysis. The first is that as temperature increases, the resonances of the low-order cladding modes from LPFGs with periods of $240,325,400$ and $550 \mu \mathrm{m}$ blue-shift towards shorter wavelengths, showing possessing negative thermal coefficients, whereas the resonances of the high-order modes of the LPFGs with periods of 110, 78 and $34 \mu \mathrm{m}$ red-shift towards longer wavelengths, corresponding to the positive thermal coefficients. Secondly, the thermal-induced resonance shift rate increases as the mode order increases in the blue-shift regime, but it decreases in the red-shift regime. Apart from the $240 \mu \mathrm{m}$ LPFG, the thermal responses of all the other six gratings exhibit a linear characteristic. We have evaluated the temperature sensitivity $\left(\Delta \lambda_{\text {res }} / \Delta \boldsymbol{T}\right)$ for each grating using the linear regression fitting technique and the results are also listed in table 1. It is evident from table 1 that the LPFGs written in the B/Ge fiber have achieved, on average, one order of magnitude higher sensitivity than that of the LPFGs produced in the standard telecom fiber. However, the maximum sensitivity of $1.75 \mathrm{~nm} /{ }^{\circ} \mathrm{C}$ achieved by the $10^{\text {th }}$ cladding mode of the $240 \mu \mathrm{m}$ LPFG is nearly 24 times that of the minimum 
value $(0.075 \mathrm{~nm} / \mathrm{C})$ exhibited by the $30^{\text {th }}$ mode of the $34 \mu \mathrm{m}$ LPFG. This clearly indicates that the thermal response of the LPFGs is strongly dependent on the order of the cladding mode.

Although the thermal response of the $240 \mu \mathrm{m}$ LPFG over the entire temperature range $\left(10-60^{\circ} \mathrm{C}\right)$ is highly non-linear, the temperature sensitivity achieved by this grating is super-high. This suggests that the 10th cladding mode of this grating belongs to the sensitive regime. In the experiment, this grating was carefully re-characterised for the temperature range just from $0^{\circ} \mathrm{C}$ to $10^{\circ} \mathrm{C}$. A total of $-27.5 \mathrm{~nm}$ wavelength shift was obtained for such a small thermal tuning range, achieving an sensitivity of $2.75 \mathrm{~nm} /{ }^{\circ} \mathrm{C}$, which is notably higher than its average value of $1.75 \mathrm{~nm} /{ }^{\circ} \mathrm{C}$, and even more than three times that of the LPFGs with the sensitivity enhanced by the special polymers [3,4]. The fact that such a large wavelength shift obtainable over such a small temperature range could lead to realisation of practical LPFG-based broadband tunable loss filters of high tuning efficiency using low-cost electric/thermal controller. It is also worthy to pointed out that if such an LPFG is use as thermal sensor interrogated by a $0.1 \mathrm{~nm}$-resolution OSA, the sensitivity of $2.75 \mathrm{~nm} /{ }^{\circ} \mathrm{C}$ could offer a temperature resolution as high as $0.036^{\circ} \mathrm{C}$.

In order to explain the non-linear thermal response of the super-sensitive LPFG, we have calculated $d \lambda_{\text {res }} / d T$ as a function of the mode order for three different resonance wavelengths $-1450 \mathrm{~nm}, 1500 \mathrm{~nm}$ and $1550 \mathrm{~nm}$ - and the results are presented in fig.3. Clearly, the $10^{\text {th }}$ and $11^{\text {th }}$ modes are the most sensitive modes, but $\left|\boldsymbol{d} \lambda_{\text {res }} / \boldsymbol{d T}\right|$ increases for the $10^{\text {th }}$ mode whereas decreases for the $11^{\text {th }}$ mode when the wavelength increases. As a result, when the $10^{\text {th }}$ mode blue-shifts as temperature increases, we expect the blue-shift rate decreases, which is again in excellent agreement with the observation in the experiment.

\section{CONCLUSIONS}

In summary, we have theoretically and experimentally investigated the thermal properties of the LPFGs which were UV written in the conventional B/Ge co-doped fiber. It has been identified that the temperature sensitivity of LPFGs can be remarkably enhanced by utilising $\mathrm{B} / \mathrm{Ge}$ fiber and choosing resonant cladding modes associated with the sensitive regime. A maximum of wavelength shift of $27.5 \mathrm{~nm}$ has been achieved from only $10^{\circ} \mathrm{C}$ change in temperature by an LPFG of $240 \mu \mathrm{m}$ period. Such a large wavelength shift obtainable over such a small temperature range could lead to realisation of practical LPFG-based devices, such as broadband tunable loss filters of high tuning efficiency at very low costs. We also anticipate that such LPFGs could be use as thermal sensors capable of extremely high temperature resolution. For example, if a $0.1 \mathrm{~nm}$-resolution OSA is used to detect temperature encoded wavelength shift, the sensitivity of $2.75 \mathrm{~nm} /{ }^{\circ} \mathrm{C}$ could offer a temperature resolution as high as $0.036^{\circ} \mathrm{C}$.

\section{ACKNOLEDGEMENTS}

This work was carried out with support of the UK EPSRC research grant.

\section{REFERENCES}

1. A.M. Vengsarkar, P.J. Lemaire, J.B. Judkins, V. Bhatia, T. Erdogan, and J.E. Sipe, "Long-period fiber gratings as band-rejection filters," J. Lightwave Technol., vol. 14, no. 1, pp. 58-64, 1996.

2. V.Bhatia and A.M. Vengsarkar, "Optical fiber long-period grating sensors," Opt. Lett., vol. 21, no. 9, pp.692-694, 1996. 
3. A.A. Abramov, B.J. Eggleton, J.A. Rogers, R.P. Espindola, A. Hale, R.S. Windeler, and T.A. Strasser, "Electrically tunable efficient broad-band fiber filter," IEEE Photon. Technol. Lett., vol.11, no. 4, pp. 445-447, 1999.

4. A.A. Abramov, A. Hale, R.S. Windeler, and T.A. Strasser, "Widely tunable long-period fiber gratings," Electron. Lett., vol. 35 , no. 1 , pp. 81-82, 1999.

5. C.G. Atherton, A.L. Steele, and J.E. Hoad, "Resonance conditions of long-period gratings in temperature sensitive polymer ring optical fiber," IEEE Photon. Technol. Lett., vol.12 no. 1, pp. 65-67, 2000.

6. M.J. Adams, An introduction to optical waveguides, John Weley \& Sons, Chichester, 1981.

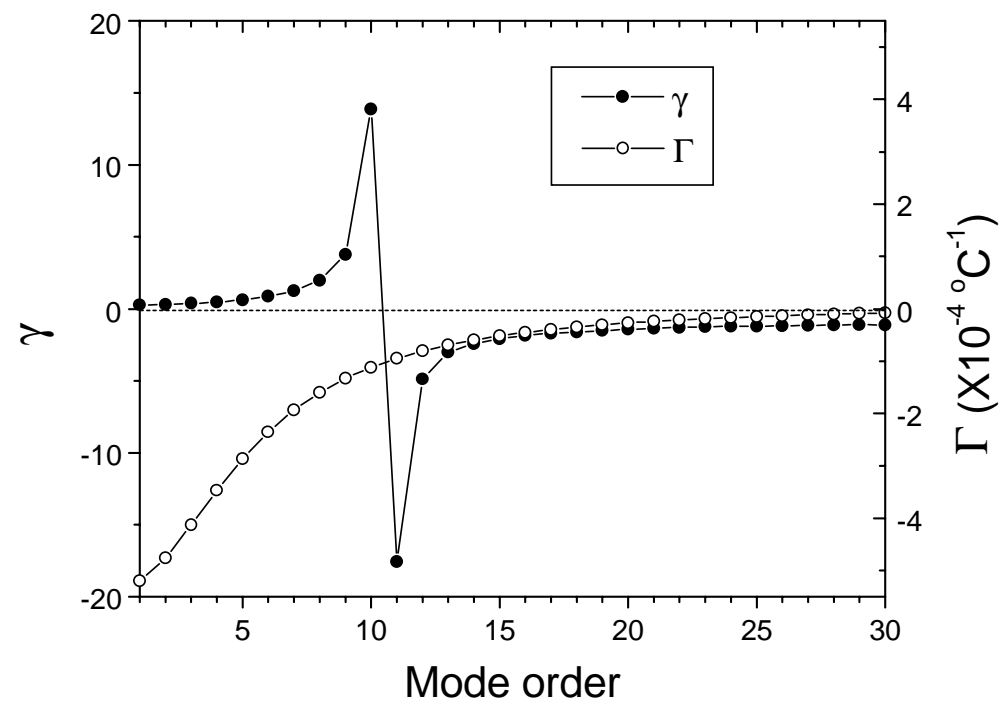

Fig.1 Calculated $\gamma$ and $\Gamma$ for the first thirty resonant cladding modes in $\mathrm{B} / \mathrm{Ge}$ codoped fiber.

Table 1

Characteristics of LPFGs fabricated in B/Ge codoped photosensitive fiber.

\begin{tabular}{|c|c|c|c|}
\hline$\Lambda(\mu \mathrm{m})$ & $\lambda$ res $(\mathrm{nm})$ & Mode order & $\mathrm{d} \lambda / \mathrm{dT}(\mathrm{nm} / \mathrm{C})$ \\
\hline 550 & 1492.4 & 5 & -0.369 \\
400 & 1524.0 & 7 & -0.493 \\
325 & 1467.0 & 8 & -0.579 \\
240 & 1530.1 & 10 & $-1.75^{*}$ \\
110 & 1523.8 & 16 & +0.377 \\
78 & 1442.7 & 20 & +0.212 \\
34 & 1502.0 & 30 & +0.075 \\
\hline
\end{tabular}

*Averaged value in the range of $0-60^{\circ} \mathrm{C}$ 


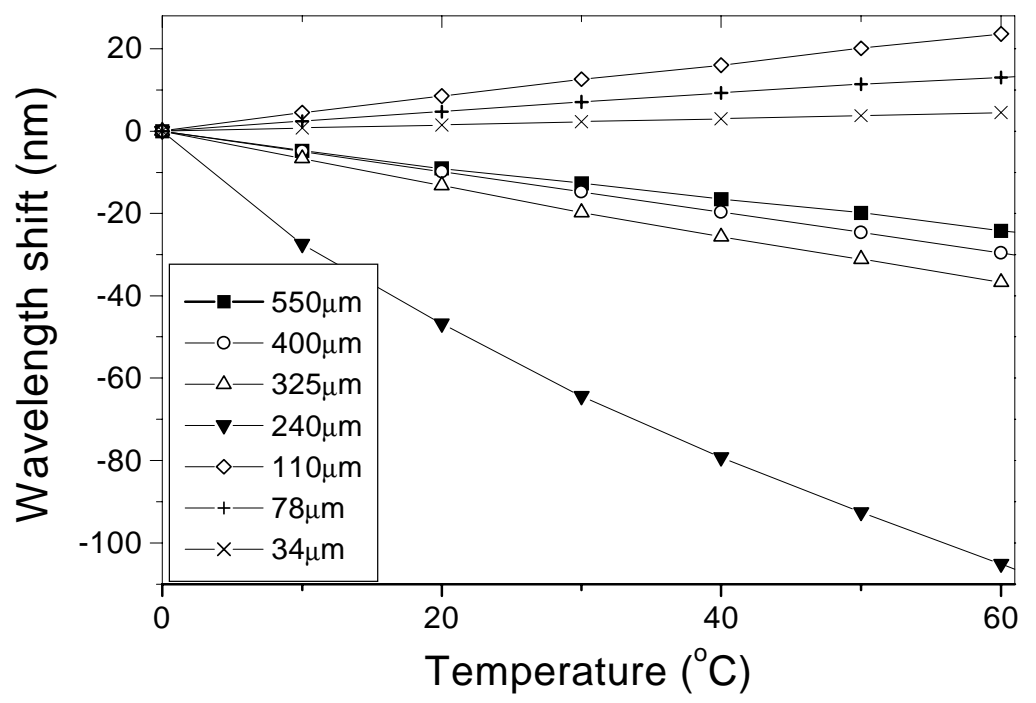

Fig.2 Measured wavelength shifts of LPFGs with temperature for different grating periods.

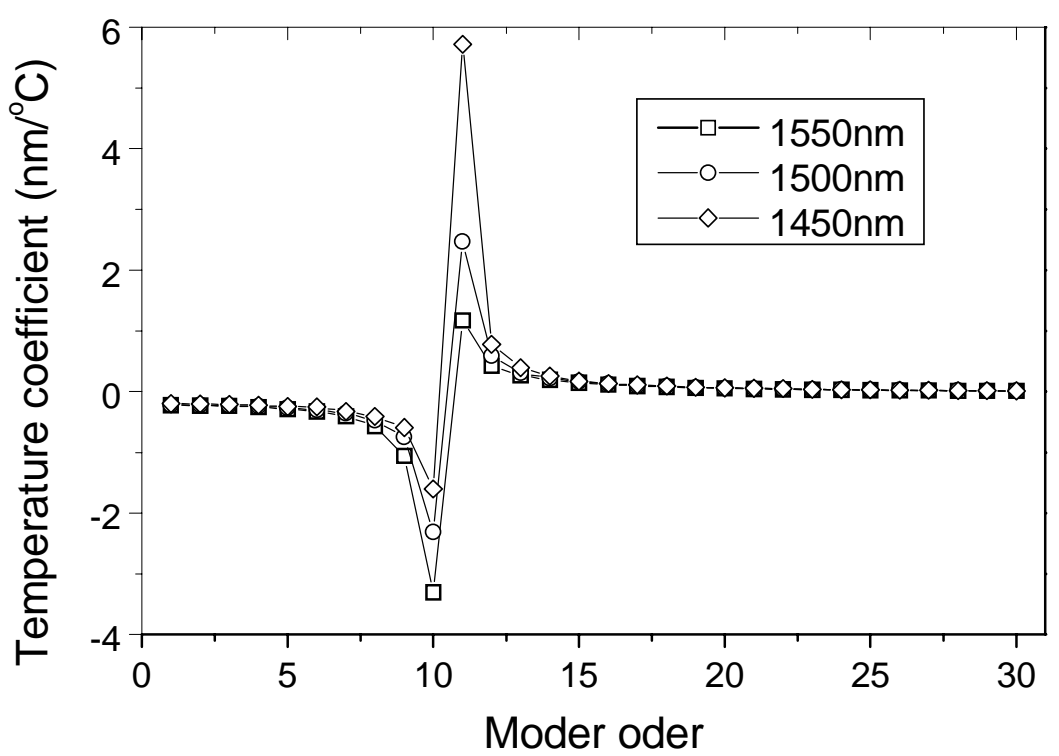

Fig. 3 Calculated temperature coefficients for the first thirty resonant cladding modes at different resonant wavelengths in $\mathrm{B} / \mathrm{Ge}$ codoped fiber. 